\title{
Sign-Compatibility of Some Derived Signed Graphs
}

Deepa Sinha* and Ayushi Dhama†

\section{Abstract}

\begin{abstract}
A signed graph (or sigraph in short) is an ordered pair $S=\left(S^{\mathcal{u}}, \sigma\right)$, where $S^{\mathcal{u}}$ is a graph $G=(V, E)$, called the underlying graph of $S$ and $\sigma: E \rightarrow\{+1,-1\}$ is a function from the edge set $E$ of $S^{\mathcal{U}}$ into the set $\{+1,-1\}$, called the signature of $S$. A sigraph $S$ is sign-compatible if there exists a marking $\mu$ of its vertices such that the end vertices of every negative edge receive ' -1 ' marks in $\mu$ and no positive edge does so. In this paper, we characterize $S$ such that its $x$-line sigraphs, semi-total line sigraphs, semi-total point sigraphs and total sigraphs are sign-compatible.
\end{abstract}

Keywords: Sign-compatible, x-line sigraph, semi-total line sigraph, semi- total point sigraph, total sigraph.

\section{Introduction}

For standard terminology and notation in graph theory we refer to Harary [6] and West [13] and Zaslavsky [14, 15] for sigraphs. Throughout the text, we consider finite, undirected graph with no loops or multiple edges.

*Department of Mathematics, South Asian University, Akbar Bhawan, Chanakyapuri, New Delhi-110 021, India; deepa_sinha2001@yahoo.com

† Centre for Mathematical Sciences, Banasthali University, Banasthali304022, Rajasthan, India; ayushi.dhama2@gmail.com

Received: July 2012, Reviewed: Aug. 2012 
A signed graph (or sigraph in short; see [5]) is an ordered pair $S=\left(S^{\mathcal{u}}, \sigma\right)$, where $S^{\mathcal{u}}$ is a graph $G=(V, E)$, called the underlying graph of $S$ and $\sigma: E \rightarrow\{+1,-1\}$ is a function from the edge set $E$ of $S^{\mathcal{U}}$ into the set $\{+1,-1\}$, called the signature of $S$. Let $E^{+}(S)=\{e \in E(G): \sigma(e)=+1\}$ and $E^{-}(S)=\{e \in E(G): \sigma(e)$ $=-1\}$. The elements of $E^{+}(S)$ and $E^{-}(S)$ are called positive and negative edges of $S$, respectively. A sigraph is all-positive (allnegative) if all its edges are positive (negative); further, it is said to be homogeneous if it is either all-positive or all-negative and heterogeneous otherwise. The positive (negative) degree of a vertex $v \in V(S)$ denoted by $d^{+}(v)\left(d^{-}(v)\right)$ is the number of positive (negative) edges incident on the vertex $v$ and $d(v)=d^{+}(v)+d^{-}(v)$.

A marked sigraph is an ordered pair $S_{\mu}=(S, \mu)$ where $S=\left(S^{u}, \sigma\right)$ is a sigraph and $\mu: V(S) \rightarrow\{+1,-1\}$ is a function from the vertex set $V(S)$ of $S$ into the set $\{+1,-1\}$, called a marking of $S$.

For a sigraph $S$, Behzad and Chartrand [1] defined its line sigraph, $L(S)$ as the sigraph in which the edges of $S$ are represented as vertices, two of these vertices are defined adjacent whenever the corresponding edges in $S$ have a vertex in common, any such edge ef is defined to be negative whenever both $e$ and $f$ are negative edges in $S$.

For a sigraph $S$, Gill [4] defined its $\times$-line sigraph $L \times(S)$ as follows: the $L \times(S)$ is a sigraph defined on the line graph $L\left(S^{\mathcal{u}}\right)$ of the graph $S^{\mathcal{U}}$ by assigning to each edge ef of $L\left(S^{\mathcal{U}}\right)$, the product of signs of the adjacent edges $e$ and $f$ of $S$.

The semi-total line graph $T_{1}(G)$ [7] of a graph $G$ is the graph whose vertex set is $V(G) \cup E(G)$ where $V(G)$ and $E(G)$ are vertex set and edge set of $G$, respectively and in $T_{1}(G)$ two vertices are adjacent if and only if (i) they are adjacent edges in $G$, or (ii) one is a vertex and the other is an edge in $G$ incident to it.

The semi-total point graph $T_{2}(G)$ [7] of a graph $G$ is the graph whose vertex set is $V(G) \cup E(G)$ where $V(G)$ and $E(G)$ are vertex set 
and edge set of $G$ respectively and in $T_{2}(G)$ two vertices are adjacent if and only if (i) they are adjacent vertices in $G$, or (ii) one is a vertex and the other is an edge in $G$ incident to it.

Let $S=(V, E, \sigma)$ be any sigraph. Its semi-total line sigraph $T_{1}(S)$ [as shown in Figure 1] has $T_{1}\left(S^{\mathcal{U}}\right)$ as its underlying graph and for any edge $u v$ of $T_{1}\left(S^{\mathcal{u}}\right)$

$$
\sigma_{T_{1}}(u v)=\left\{\begin{array}{l}
\sigma(u) \sigma(v) \text { if } u, v \in E \\
\sigma(u) \text { if } u \in E \text { and } v \in E .
\end{array}\right.
$$

Let $S=(V, E, \sigma)$ be any sigraph. Its semi-total point sigraph $T_{2}(S)$ [as shown in Figure 1] has $T_{2}\left(S^{\mathcal{u}}\right)$ as its underlying graph and for any edge $u v$ of $T 2\left(S^{\mathcal{u}}\right)$

$$
\sigma_{T_{2}}(u v)=\left\{\begin{array}{l}
\sigma(u v) \text { if } u, v \in E \\
\sigma(u) \prod_{e_{j} \in E_{v}} \sigma\left(e_{j}\right) \text { if } u \in E \text { and } v \in E .
\end{array}\right.
$$

We observe that $L_{\times}(S)$ is an induced subsigraph of $T_{1}(S)$ and $S$ is an induced subsigraph of $T_{2}(S)$.

The total graph $T(G)[2,3]$ of a graph $G$ is that graph whose vertex set is $V(G) \cup E(G)$ where $V(G)$ and $E(G)$ are vertex set and edge set of $G$ respectively and in $T(G)$ two vertices are adjacent if and only if they are adjacent or incident in $G$.

Let $S=(V, E, \sigma)$ be any sigraph. Its total sigraph $T(S)$ [as shown in Figure 1] has $T\left(S^{\mathcal{U}}\right)$ as its underlying graph and for any edge $u v$ of $T\left(S^{u}\right)$ the sign of the edge is defined as (i) if $u, v \square V$, then $\sigma_{T}(u v)=\sigma(u v)$ (ii) if $u, v \square E$, then $\sigma_{T}(u v)=\sigma(u) \sigma(v) \quad$ (iii) if $u \square E$ and $v \square V$, then $\sigma_{T}(u v)=\sigma(u) \prod e_{j} \in E_{v} \sigma\left(e_{j}\right)$.

We observe that $S$ and $L \times(S)$ are the induced subsigraphs of $T(S)$. Also, $T_{2}(S)$ is a subsigraph of $T(S)$. Much of the work has already been done on the structures $T_{1}(S), T_{2}(S)$ and $T(S)$. (see $[9,10,11])$ 
Looking into the structures of the line sigraphs it was observed [8] that the vertices of a line sigraph can be marked so that both the ends of every negative edge receive negative marks and no positive edge receives negative marks at both of its ends. Since the converse of the above statement does not hold, as can be seen in Figure 2 and Figure 3, therefore it becomes important to investigate the notion of 'sign-compatibility' in sigraphs.

A sigraph $S$ is sign-compatible [8] if there exists a marking $\mu$ of its vertices such that the end vertices of every negative edge receive ' -1 ' marks in $\mu$ and no positive edge in $S$ has both of its ends assigned ' -1 ' marks in $\mu$, sign-incompatible otherwise.

Theorem 1. [8] A sigraph $S$ is sign-compatible if and only if its vertices can be partitioned into two subsets $V_{1}$ and $V_{2}$ such that the all-negative subsigraph of $S$ is precisely the subsigraph induced by exactly one of the subset $\mathrm{V}_{1}$ or $\mathrm{V}_{2}$.
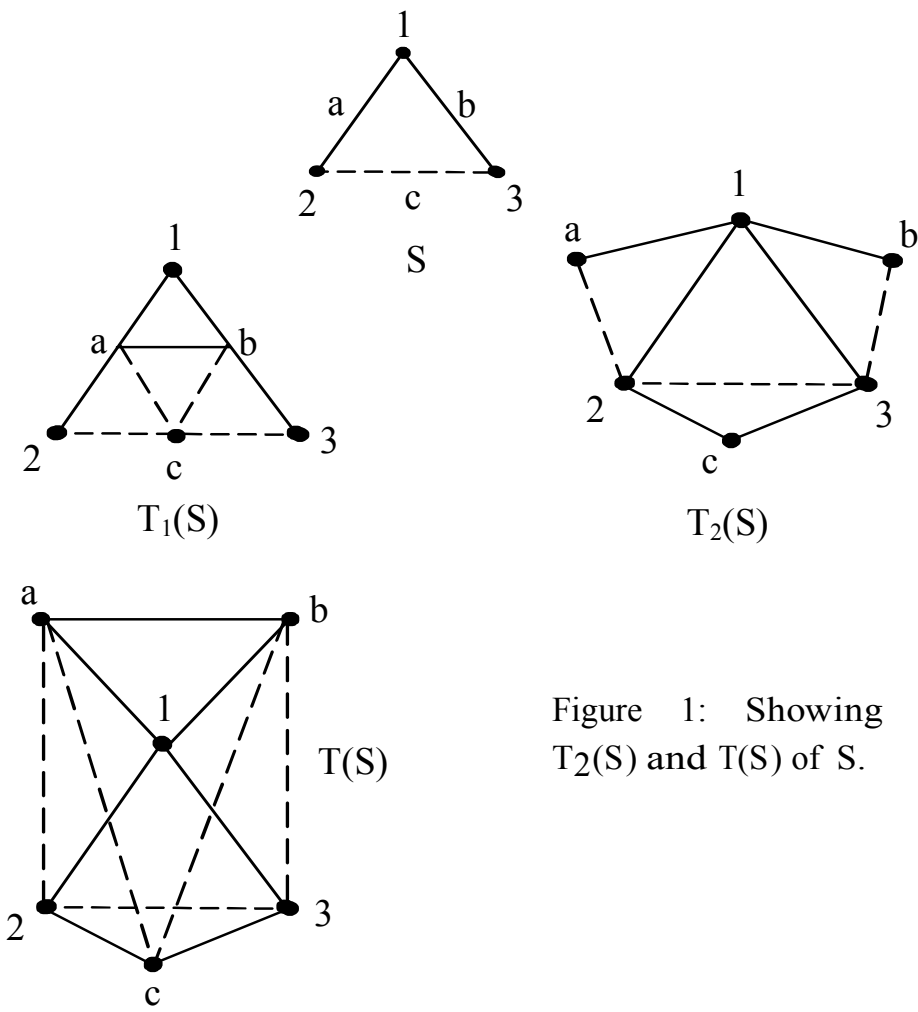

Figure 1: Showing $\mathrm{T}_{1}(\mathrm{~S})$, $\mathrm{T}_{2}(\mathrm{~S})$ and $\mathrm{T}(\mathrm{S})$ of $\mathrm{S}$. 


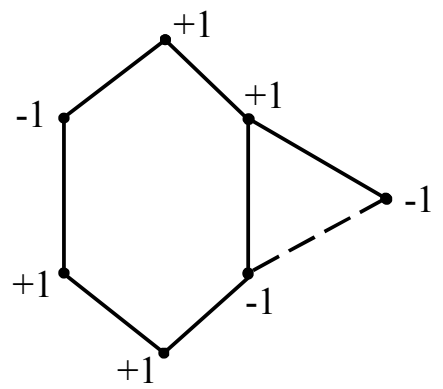

Figure 2: A line sigraph which is sign-compatible.

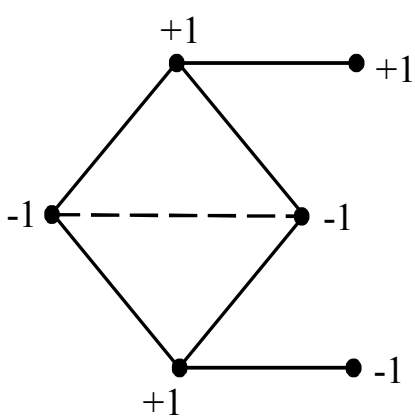

Figure 3: A sigraph which is sign-compatible but not a line sigraph.

Proposition 2. Every subsigraph of a sign-compatible sigraph is sign-incompatible.

Theorem 3. [12] A sigraph $S$ is sign-compatible if and only if $S$ does not contain a subsigraph isomorphic to either of the two sigraphs, $S_{1}$ formed by taking the path $P_{4}=(x, u, v, y)$ with both the edges $\mathrm{xu}$ and vy negative and the edge uv positive and $\mathrm{S}_{2}$ formed by taking $S_{1}$ and identifying the vertices $x$ and $y$ (Figure 4).

\section{Sign-Compatibility of $x$-line Sigraphs}

In this section we establish a characterization of signcompatible $\mathrm{x}$-line sigraphs. The observations, which becomes 
ready reference for the proof of the theorems, are stated as under.

Observation 4. For a given sigraph $\mathrm{S}=\left(\mathrm{S}^{\mathrm{u}}, \sigma\right)$, if $L_{\times}(S)$ is heterogeneous, then $S$ is heterogeneous but converse is not true, as can be seen in Figure 5.

Observation $5 . \mathrm{Lx}(\mathrm{S})$ is all-negative if and only if $\mathrm{S}$ is either an even cycle or a path with alternative ' +1 ' and ' -1 ' signs.

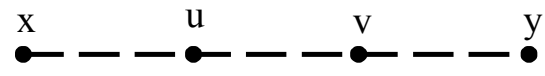

$\mathrm{S}_{1}$

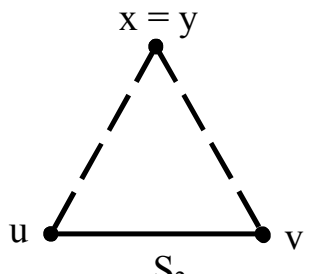

$\mathrm{S}_{2}$

Figure 4: Acharya and Sinha forbidden subsigraphs for a signcompatible sigraph
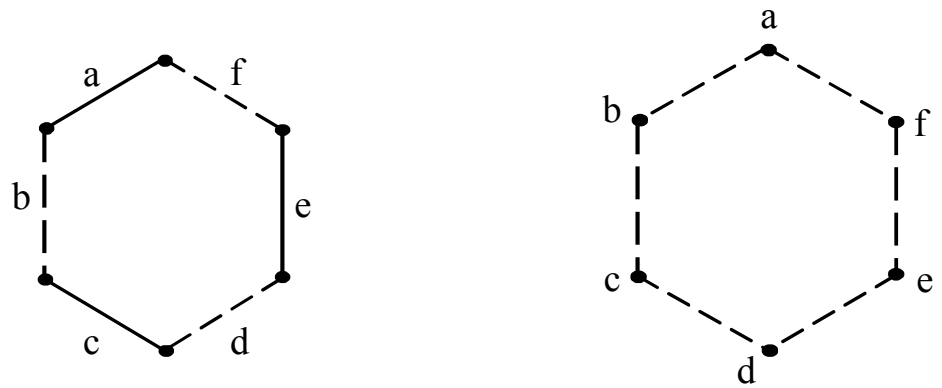

Figure 5: A sigraph and its $\mathrm{x}$-line sigraph

Observation 6. $\mathrm{L}_{\times}(\mathrm{S})$ is all-positive if and only if $\mathrm{S}$ is either all-positive or all-negative.

The following theorem determines the solution when a $x$-line sigraph is sign- compatible.

Theorem 7. For a given sigraph $S=\left(S^{\mathrm{u}}, \sigma\right), L_{\times}(S)$ is signcompatible if and only if for any two adjacent positive (negative) edges, say $e_{i}$ and $e_{j}$, either there is no negative (positive) edge 6 
adjacent with $e_{i}$ or there is no negative (positive) edge adjacent with ej in $S$.

Proof. Necessity: Suppose $L \times(S)$ is sign-compatible. Then, by Theorem $3, L \times(S)$ does not contain a subsigraph isomorphic to either $S_{1}$ or $S_{2}$ in Figure 4.

Let there be two adjacent positive (negative) edges, say $e_{i}$ and $e_{j}$, in $S$ and there are negative (positive) edges adjacent with $e_{i}$ and $e_{j}$. Either there is one negative (positive) edge, which is adjacent with both $e_{i}$ and $e_{j}$ or there are distinct negative (positive) edges adjacent with $e_{i}$ and $e_{j}$. If there is one negative (positive) edge adjacent with both $e_{i}$ and $e_{j}$, then by the definition of $L \times(S)$, $L \times(S)$ contains a subsigraph isomorphic to $S_{2}$, a contradiction to our assumption. Now, let $e_{k}$ be a negative (positive) edge adjacent with $e_{i}$ and $e l$ is a negative (positive) edge adjacent with $e_{j}$ in $S$, then by the definition of $L \times(S)$, we have a path $P_{4}=\left(e_{k}, e_{i}, \mathrm{e}_{j}, e_{l}\right)$ in $L \times(S)$ such that $e_{k} e_{i}$ and $e_{j} e_{l}$ are negative edges and $e_{i} e_{j}$ is a positive edge. Thus, $L \times(S)$ contains a subsigraph isomorphic to $S_{1}$, a contradiction to our assumption. Thus, the condition follows.

Sufficiency: Suppose condition in the statement of the theorem holds for a sigraph $S$. We want to show that $L \times(S)$ is sign-compatible. Let on contrary, $L \times(S)$ is not sign-compatible. Then, by Theorem 3, $L \times(S)$ contains a subsigraph isomorphic to either $S_{1}$ or $S_{2}$.

Case I: Let $L \times(S)$ contains a subsigraph, say $P 4^{\prime}$, isomorphic to $S_{1}$. Let $P_{4}=\left(e_{\mathbf{i}}, e_{j}, e_{k}, e_{l}\right)$ such that $e_{i} e_{j}$ and $e_{k} e_{l}$ are negative edges and $e_{j} e_{k}$ is a positive edge in $L \times(S)$. Then, by the definition of $L \times(S)$, we have a path $P_{5}=(u, v, w, x, y)$ in $S$ such that $e_{i}=u v, e_{j}=v w, e_{k}=w x$ and $e_{l}=x y$. Also, $e_{j}, e_{k}$ are positive (negative) edges and $e_{i}, e l$ are negative (positive) edges in $S, a$ contradiction to the hypothesis. Thus, $L \times(S)$ does not contain a subsigraph isomorphic to $S 1$. 
Case II: Now, let $L \times(S)$ contains a subsigraph isomorphic to $S_{2}$, then this triangle is either due to the edges of a triangle or due to a vertex $v \in V(S)$ in $S$ with $d(v) \geq 3$.

II (a): Let $Z^{\prime}$ be a triangle in $L \times(S)$ which is isomorphic to $S_{2}$. If all the vertices of $Z^{\prime}$ are due to the adjacent edges of a single triangle $Z$ in $S$, then by the definition of $L \times(S)$, we have a triangle $Z$ in $S$ with two positive (negative) and one negative (positive) edges. Thus, for two adjacent positive (negative) edges in $S$, we have a negative (positive) edge adjacent with these edges, a contradiction to hypothesis.

II (b): Now, since this triangle is not due to any triangle of $S$, therefore $Z^{\prime}$ must contain a vertex, say $e_{p}$, which corresponds to an edge $e_{p}$ not lying on any cycle but incident to a vertex $v$ with $d(v) \geq 3$ in $\mathrm{S}$. Then, by the definition of $L \times(S)$, there are two positive (negative) and one negative (positive) edges incident on $v$ in $S$. Thus, we have a contradiction to the hypothesis. Hence, $L \times(S)$ does not contain a subsigraph isomorphic to $S_{2}$. Hence, by Theorem $3, L \times(S)$ is sign- compatible.

Note: In the all upcoming theorems, ei,ej,ek, el and em denote the edges of $S$ and $v i, v j, v k, v l, v m$ and $v n$ denote the vertices of $S$.

\section{Sign-compatibility of Semi-total Line Sigraphs}

Next, for studying the characterization of yet another structure, semi-toatl line sigraphs, we have the following observation.

Observation 8. For a given sigraph $S=\left(S^{\mathcal{u}}, \sigma\right), T_{1}(S)$ is never all-negative.

The following theorem determines the solution when a semi-total line sigraph is sign-compatible.

Theorem 9. For a given sigraph $S=\left(S^{\mathcal{u}}, \sigma\right), T_{1}(S)$ is signcompatible if and only if $S$ is all-positive. 
Proof. Necessity: Let $T_{1}(S)$ be sign-compatible. Then, by Theorem 3, $T_{1}(S)$ does not contain a subsigraph isomorphic to either $S_{1}$ or $S_{2}$.

Let $e_{l}=v_{i} v_{j}$ and $e_{m}=v_{j} v_{k}$ be two adjacent edges in $S$. If one is positive and other is negative or both are negative, then by the definition of $T_{1}(S)$, we have a triangle $\left(e_{l}, e_{m}, v_{j}, e_{l}\right)$ in $T_{1}(S)$ with one positive and two negative edges. Thus, $T_{1}(S)$ contains a subsigraph isomorphic to $S_{2}$, a contradiction to the hypothesis. Hence, no two adjacent edges can be heterogeneous or both negative in $S$. Hence, $S$ is all-positive.

Sufficiency: Let $S$ be all-positive, then by the definition of $T_{1}(S), T_{1}(S)$ is also all-positive. Hence, $T_{1}(S)$ is sign-compatible.

\section{Sign-compatibility ofSsemi-total Point Sigraphs}

With the Observation 10 and Observation 11, we now venture for the characterization of sign-compatible semi-total pointsigraphs.

Observation 10. For a given sigraph $S=\left(S^{\mathcal{u}}, \sigma\right)$, if $S$ is heterogeneous then $T_{2}(S)$ is also heterogeneous but converse is not true, as can be seen in Figure 6.

Observation 11. $T_{2}(S)$ is all-negative if and only if $S$ is allnegative and $d(v)$ is even $\forall v \in V(S)$.

The following theorem determines the solution when a semi-total point sigraph is sign-compatible.

Theorem 12. For a given sigraph $S=\left(S^{\mathcal{u}}, \sigma\right), T_{2}(S)$ is signcompatible if and only if for any edge $e_{k}=v_{i} v_{j}$ in $S$, 


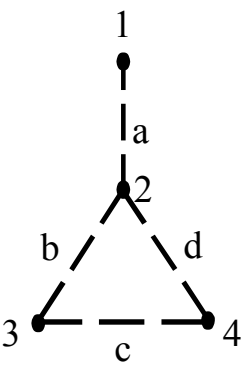

S

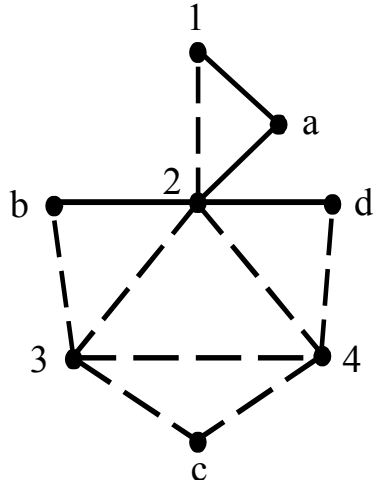

$T_{2}(S)$

Figure 6: A sigraph and its semi-total point sigraph.

i. if $e_{k}$ is a positive edge, then either there is no negative edge on $v_{i}$ or there is no negative edge on $v_{j}$ and

ii. if $e_{k}$ is a negative edge, then $d^{-}\left(v_{i}\right)$ and $d^{-}\left(v_{j}\right)$ are of same parity.

Proof. Necessity: Let $T_{2}(S)$ be sign-compatible. Then, by Theorem 3, T2 $(S)$ does not contain a subsigraph isomorphic to either $S_{1}$ or $S_{2}$.

Let $e_{k}=v_{i} v_{j}$ be any edge in $S$. First we assume that $e_{k}$ is a positive edge. Let for a positive edge $e_{k}=v_{i} v_{j}$, there be negative edges on $v_{i}$ and $v_{j}$. This implies that $S$ contains a subsigraph isomorphic to either $S_{1}$ or $S_{2}$. Thus, $T_{2}(S)$ contains a subsigraph isomorphic to either $S_{1}$ or $S_{2}$, a contradiction to our assumption. Hence, (i) follows.

Next, let $e_{k}=v_{i} v_{j}$ be negative edge in $S$ and $d^{-}\left(v_{i}\right)$ and $d^{-}\left(v_{j}\right)$ are of opposite parity i.e., $d^{-}\left(v_{i}\right)$ is odd and $d^{-}\left(v_{j}\right)$ is even or vice-versa. Then, by the definition of $T_{2}(S)$, we have a triangle $\left(v_{i}\right.$ $\left., v_{j}, e_{k}, v_{i}\right)$, with one positive and two negative edges in $T_{2}(S)$. Thus, $T 2(S)$ contains a subsigraph isomorphic to $S_{2}$, a contradiction to our assumption. Hence, (ii) follows. 
Sufficiency: Suppose conditions (i) and (ii) hold for a given sigraph $S$. We shall show that $T_{2}(S)$ is sign-compatible. Suppose on contrary, $T_{2}(S)$ is not sign-compatible. Then, by Theorem 3, $T_{2}(S)$ contains a subsigraph isomorphic to either $S_{1}$ or $S_{2}$.

Case I: Let $T 2(S)$ contains a subsigraph, say $P 4$, isomorphic to S1. Now, $P_{4}$ will be one out of $\left(v_{i}, v_{j}, v_{k}, v_{l}\right),\left(v_{i}, v_{j}, v_{k}, e_{l}\right)$, $\left(e_{i}, v_{j}, v_{k}, e_{l}\right),\left(v_{i}, v_{j}, e_{k}, v_{l}\right)$ and $\left(e_{i}, v_{j}, e_{k}, v_{l}\right)$. Here $P_{4}=\left(v_{i}, v_{j}, v_{k}, v_{l}\right)$, otherwise we have a contradiction to (i).

I(a): Let $P_{4}=\left(v_{i}, v_{j}, e_{k}, v_{l}\right)$ or $\left(e_{i}, v_{j}, e_{k}, v_{l}\right)$. In both the cases $e_{k}$ is not a negative edge in $S$ otherwise we have a subsigraph, $\left(v_{j}, e_{k}, v_{l}, v_{j}\right)$, isomorphic to $S_{2}$ in $T_{1}(S)$. Thus, we have one negative edge, $e_{k}$, in $S$ such that the negative degree of the end vertices of $e_{k}$ have opposite parity, a contradiction to (ii). Hence, $e_{k}$ is a positive edge in $S$. When $P_{4}=\left(v_{i}, v_{j}, e_{k}, v_{l}\right)$, then $e_{k} v_{l}$ is a negative edge in $T 2(S)$, then by the definition of $T 2(S)$, there are odd no of negative edges incident on $v$ in $S$. Thus, we have a positive edge $e_{k}=v_{j} v_{l}$ in $S$ such that there are negative edges incident on $v j$ and $v l$, a contradiction to (i).

Now, when $P_{4}=\left(e_{i}, v_{j}, e_{k}, v_{l}\right)$ either $e_{i}$ is a negative or a positive edge in $S$, but in both the cases, by the definition of $T_{2}(S)$, we have a positive edge $e_{k}=v_{j} v_{l}$ in $S$ such that there are negative edges incident on $v_{j}$ and $v_{l}$, a contradiction to (i).

I(b): Let $P_{4}=\left(v_{i}, v_{j}, v_{k}, e_{l}\right)$ or $P_{4}=\left(e_{i}, v_{j}, v_{k}, e_{l}\right)$, in both the cases whatever be the sign of $e_{i}$ and $e_{l}$ in $S$, by the definition of $T_{2}(S)$, we have a positive edge $v_{j} v_{k}$ in $S$ such that there are negative edges incident on $v_{j}$ and $v_{k}$, a contradiction to (i). Thus, $T_{2}(S)$ does not contain a subsigraph isomorphic to $S 1$.

Case II: Now, let $T_{2}(S)$ contains a subsigraph isomorphic to $S_{2}$. Then, either this triangle is due to the vertices of a triangle of $S$ or due to two adjacent vertices of $S$. 
II (a): Let $Z^{\prime}$ be a triangle isomorphic to $S_{2}$ in $T_{2}(S)$ and this is due to the vertices of a triangle of $S$. Then, we have a triangle with one positive and two negative edges in $S$, a contradiction to (i).

II (b): Let $Z^{\prime}$ be due to two adjacent vertices, say $v i$ and $v j$, in $S$ and let $e k=v i v j$. Now, either $e k$ is a positive or negative edge in $S$. If $e k$ is a positive edge, then by the definition of $T_{2}(S)$, we have a positive edge $e k=v i v j$ in $S$ such that there are odd number of negative edges on both the vertices, $v i$ and $v j$. This contradicts (i). If $e k$ is a negative edge in $S$, then by the definition of $T_{2}(S)$, we have a negative edge $e k$ in $S$ such that $d-(v i)$ and $d-(v j)$ are of opposite parity. This contradicts (ii). Thus, $T_{2}(S)$ does not contain a sub-sigraph isomorphic to $S_{2}$. Hence, $T_{2}(S)$ is signcompatible.

\section{Sign-compatibility of Total Sigraphs}

Having characterized about semi-total line sigraphs and semitotal point sigraphs, the natural thing that arises in ones mind is that what if both $S$ and x-line sigraph lie as an induced subsigraph in some sigraph. Yes, when the sigraph is a total sigraph. Now, we present a characterization of sign-compatible total sigraphs with the help of Observation 13.

Observation 13. $T(S)$ is never all negative.

The above observation easily follows from the fact that $S$ and $L \times(S)$ are the induced subsigraphs of $T(S)$.

The following theorem determines the solution when a total sigraph is sign-compatible.

Theorem 14. For a given sigraph $S=\left(S^{\mathcal{u}}, \sigma\right), T(S)$ is signcompatible if and only if $S$ is either all-positive or all-negative such that $d(v)$ is odd $\forall v \in V(S)$. 
Proof. Necessity: Let $T(S)$ be sign-compatible. Then, by Theorem 3, $T(S)$ does not contain a subsigraph isomorphic to either $S_{1}$ or $S_{2}$.

Now, either $T(S)$ is homogeneous or heterogeneous. By Observation 13, $T(S)$ will be either all-positive or heterogeneous. Since $S$ is an induced subsigraph of $T(S)$, therefore if $T(S)$ is allpositive, then $S$ is also all-positive. If $T(S)$ is heterogeneous, then either $S$ is heterogeneous or $S$ is all-negative. Let $e_{i}$ and $e_{j}$ be two edges of opposite signs in $S$ and incident on a vertex, say $v k$. Then, by the definition of $T(S)$, we have a triangle $\left(e_{i}, e_{j}, v_{k}, e_{i}\right)$ in $T(S)$ which is isomorphic to $S_{2}$. Thus, we get a contradiction to our assumption. Hence, $T(S)$ is not heterogeneous.

Next, let $S$ be all-negative. Since $T_{2}(S)$ is a subsigraph of $T(S)$, therefore $T_{2}(S)$ is also all-negative. Now, by Proposition 2 and Theorem 12, degree of every vertex of $T(S)$ is either odd or even. Suppose degree of any vertex, say $v_{k}$, is even in $S$. Let $e_{i}$ and $e_{j}$ be two adjacent edges on the vertex $v_{k}$. Then, by the definition of $T(S)$, we have a triangle $\left(e_{i}, e_{j}, v_{k}, e_{i}\right)$ with one positive and two negative edges. Thus, $T(S)$ contains a subsigraph isomorphic to $S_{2}$, a contradiction to our assumption. Hence, $S$ is all-negative such that $d(v)$ is odd $\forall v \in V(S)$.

Sufficiency: Suppose the condition in the theorem holds for a given sigraph $S$. If $S$ is all-positive, then by the definition of $T(S)$, $T(S)$ is also all-positive. Hence $T(S)$ is sign-compatible.

Now, let $S$ be all-negative such that degree of every vertex is odd. By the definition of $T(S)$, any positive edge in $T(S)$ will be either due to two adjacent edges in $S$ or due to a vertex and an edge incident on it and a negative edge in $T(S)$ is only due to two adjacent vertices i.e., any positive edge is of the form $e_{i} e_{j}$ or $v_{l} e_{k}$ and negative edge is of the form $v_{m} v_{n}$. Thus, for a positive edge in $T_{2}(S)$, there are not negative edges incident on both its end vertices. Thus, $T(S)$ does not contain a subsigraph isomorphic to either $S_{1}$ or $S_{2}$. Thus, by Theorem $3, T(S)$ is signcompatible. Hence the theorem. 


\section{Acknowledgement}

The authors express gratitude to Mr. Pravin Garg who was always there in the prior discussion of the paper to give his comments. The research is supported by Department of Science and Technology (Govt. of India), New Delhi, India under the Project SR/S4/MS: 409/06.

\section{References}

[1] M Behzad and G T Chartrand, "Line coloring of signed graphs," Elem. Math., vol. 24, pp. 49-52, 1969.

[2] M Behzad, "A characterization of total graphs," Proc. Amer. Math. Soc., vol. 26, pp. 383-389, 1970.

[3] M Behzad and H Radjavi, "Structure of regular total graphs," J. Lond. Math. Soc., vol. 44, pp. 433-436, 1969.

[4] M K Gill, "Contribution to some topics in graph theory and its applications," Ph.D. Thesis, Indian Institute of Technology, Bombay, 1983.

[5] F Harary, "On the notion of balance of a signed graph," Michigan Math. J., vol. 2, pp. 143-146, 1953.

[6] F Harary, Graph theory, Massachusetts: Addison-Wesley Publ. Comp., 1969.

[7] E Sampathkumar and S B Chikkodimath, "Semitotal graphs of a graph-I," J. Karnatak Univ. Sci., vol. 18, pp. 274-280, 1973.

[8] D Sinha, "New frontiers in the theory of signed graph," Ph.D. Thesis, University of Delhi, Faculty of Technology, 2005.

[9] D Sinha and P Garg, "Balance and consistency of total signed graphs," Ind. J. Math., vol. 53, pp. 71-81, 2011.

[10] D Sinha and P Garg, "Characterization of total signed graph and semi-total signed graphs," Int. J. Contemp. Math. Sci., vol. 6, pp. 221-228, 2011.

[11] D Sinha and P Garg, "On the regularity of some signed graph structures," AKCE Int. J. Graphs Comb., vol. 8, pp. 63-74, 2011.

[12] D Sinha and A Dhama, "Sign-compatibility of common-edge sigraphs and 2-path sigraphs," Preprint.

[13] D B West, Introduction to graph theory, Prentice-Hall of India Pvt. Ltd., 1996.

[14] T Zaslavsky, "A mathematical bibliography of signed and gain graphs and allied areas," VII Edition, Electron. J. Combin., \#DS8, 1998.

[15] T Zaslavsky, "Glossary of signed and gain graphs and allied areas," II Edition, Electron. J. Combin., \#DS9, 1998. 